Supporting Information for

\title{
Temperature-Dependent Chemical Functional Group Reorientation at Silicone Surfaces
}

\author{
Canyu Cai, ${ }^{1}$, Md. Shafiul Azam, ${ }^{1}$ Dennis K. Hore ${ }^{1,2}$ \\ ${ }^{1}$ Department of Chemistry, University of Victoria, Victoria, British Columbia, V8W 3V6, Canada \\ ${ }^{2}$ Department of Computer Science, University of Victoria, Victoria, British Columbia, V8W 3P6, Canada
}

\section{S1 Temperature-dependent refractive indices}

The temperature-dependent refractive indices were used to calculate the local field factors $\left|L_{i i} L_{j j} L_{k k}\right|^{2}$ as described in previous publications. ${ }^{1,2}$ Wavelength- and temperaturedependent (real part of the) refractive index of $\mathrm{D}_{2} \mathrm{O}$ were taken from the literature. ${ }^{3,4}$

$$
n(\lambda, T)=A+B / \lambda^{2}+C \cdot T+D \cdot T / \lambda^{2}+E \cdot T^{2}+F \cdot T^{3}
$$

where $A, B, C, D, E$, and $F$ are the Cauchy coefficients

$$
\begin{aligned}
& A=1.0244 \\
& B=3329.2 \\
& C=2.6048 \times 10^{-3} \\
& D=-1.630 \\
& E=-7.248 \times 10^{-6} \\
& F=6.15 \times 10^{-9}
\end{aligned}
$$

$\lambda$ is the wavelength in microns, and $T$ is the absolute temperature in Kelvin. The imaginary part of the $\mathrm{D}_{2} \mathrm{O}$ refractive index was close to zero ${ }^{5,6}\left(\kappa=2.98 \times 10^{-8}\right.$ at $532 \mathrm{~nm}$ and similar at SFG wavelengths, $\kappa=1.416 \times 10^{-3}$ at $\left.3.33 \mu \mathrm{m}\right)$ and therefore its temperaturedependence was not a significant source of spectral intensity variation.

The temperature-dependent refractive index of silica was incorporated in our model using the expression ${ }^{7}$

$$
n^{2}-1=\sum_{i=1}^{3} \frac{a_{i}}{b_{i}^{2}-E^{2}}
$$

with

$$
\begin{aligned}
& a_{i}=a_{i 0}+a_{i 1} t+a_{i 2} t^{2} \\
& b_{i}=b_{i 0}+b_{i 1} t+b_{i 2} t^{2} .
\end{aligned}
$$

where $E$ is the photon energy in $\mathrm{eV}$, and $t$ is the temperature in degrees Celsius. Values of the parameters $a_{i n}$ and $b_{\text {in }}$ can be found in the literature. ${ }^{7}$ 
Published values of temperature-dependent refractive index for PDMS are not available. Since for polymers the refractive index and density have a near linear relationship, ${ }^{8}$ temperature-dependent density data ${ }^{9}$ was used to estimate the change in refractive index under elevated temperatures. The room temperature complex refractive index data for PDMS was taken from the literature ${ }^{10,11}$ and reduced by $5 \%$ over the range $20-70{ }^{\circ} \mathrm{C}$.

Similarly, there is no published temperature-dependent refractive index data for FC40. The datasheet lists the room temperature refractive index in the visible as $1.29 .{ }^{12}$ As there are no vibrational resonances in our frequency range of interest, we consider the dispersion to be negligible. The manufacturer $(3 \mathrm{M})$ provides an empirical relationship to estimate the density

$$
\rho=1909-2.16 T \mathrm{~kg} \cdot \mathrm{m}^{-3}
$$

where $T$ is the temperature in degrees Celsius. Over our temperature range, the density decreases by $6 \%$, and we have scaled the FC40 index accordingly.

\section{S2 SFG spectra fitting parameters}

The PDMS-FC40 spectra were fitted with two modes: the $\mathrm{CH}_{3}$ symmetric stretching mode at $2910 \mathrm{~cm}^{-1}$ and the $\mathrm{CH}_{3}$ asymmetric stretching mode at $2963 \mathrm{~cm}^{-1}$. The widths of the modes were determined to be $10 \mathrm{~cm}^{-1}$ and $15 \mathrm{~cm}^{-1}$, respectively. As no Fermi resonance was observed for FC40 spectra, the FR mode was fixed at zero amplitude. The fit amplitudes are presented in Tables $\mathrm{S} 1$ and $\mathrm{S} 2$.

Table S1: Fit amplitudes for PDMS-FC40 SSP spectra (shown in Figure 2a).

\begin{tabular}{|l|l|l|l|l|}
\hline $\begin{array}{l}\text { Temperature } \\
\boldsymbol{I}\end{array}$ & $\begin{array}{l}\mathbf{C H}_{3} \mathbf{C S} \\
\text { amplitude }\end{array}$ & $\begin{array}{l}\mathrm{CH}_{3} \text {, as } \\
\text { amplitude }\end{array}$ & $\begin{array}{l}\text { Fermi } \\
\text { amplitude }\end{array}$ & $\begin{array}{l}\text { NR } \\
\text { amplitude }\end{array}$ \\
\hline 20 & 21.55 & -12.46 & 0.00 & 0.04 \\
\hline 30 & 20.64 & -12.15 & 0.00 & 0.04 \\
\hline 40 & 19.57 & -11.88 & 0.00 & 0.04 \\
\hline 50 & 17.74 & -10.69 & 0.00 & 0.04 \\
\hline 60 & 15.67 & -10.34 & 0.00 & 0.04 \\
\hline 70 & 13.15 & -9.46 & 0.00 & 0.05 \\
\hline
\end{tabular}

Table S2: Fit amplitudes for PDMS-FC40 SPS spectra (shown in Figure 2c).

\begin{tabular}{|l|l|l|l|l|}
\hline $\begin{array}{l}\text { Temperature } \\
\boldsymbol{}^{\circ} \mathbf{C}\end{array}$ & $\begin{array}{l}\mathbf{C H}_{3} \text { SS } \\
\text { amplitude }\end{array}$ & $\begin{array}{l}\mathbf{C H}_{3} \text {, as } \\
\text { amplitude }\end{array}$ & $\begin{array}{l}\text { Fermi } \\
\text { amplitude }\end{array}$ & $\begin{array}{l}\text { NR } \\
\text { amplitude }\end{array}$ \\
\hline 20 & -0.15 & 8.43 & 0.00 & 0.26 \\
\hline 30 & -0.44 & 8.41 & 0.00 & 0.25 \\
\hline 40 & -0.58 & 7.94 & 0.00 & 0.23 \\
\hline 50 & -0.29 & 7.24 & 0.00 & 0.22 \\
\hline 60 & -0.47 & 6.93 & 0.00 & 0.20 \\
\hline 70 & -0.62 & 6.69 & 0.00 & 0.16 \\
\hline
\end{tabular}


The PDMS- $\mathrm{D}_{2} \mathrm{O}$ spectra were fit with three modes: the $\mathrm{CH}_{3}$ symmetric stretching mode at $2910 \mathrm{~cm}^{-1}$, the $\mathrm{CH}_{3}$ asymmetric stretching mode at $2963 \mathrm{~cm}^{-1}$, and a Fermi resonance at $2938 \mathrm{~cm}^{-1} \cdot{ }^{13-16}$ The width for the modes were determined to be $15 \mathrm{~cm}^{-1}$, $8 \mathrm{~cm}^{-1}$, and $15 \mathrm{~cm}^{-1}$, respectively, from initial searching using global fitting. The fit amplitudes are presented in Tables S3 and S4.

Table S3: Fit amplitudes for PDMS- $\mathrm{D}_{2} \mathrm{O}$ SSP spectra (shown in Figure 2b).

\begin{tabular}{|l|l|l|l|l|}
\hline $\begin{array}{l}\text { Temperature } \\
\boldsymbol{}^{\circ} \mathbf{C}\end{array}$ & $\begin{array}{l}\mathbf{C H}_{3} \text { Ss } \\
\text { amplitude }\end{array}$ & $\begin{array}{l}\mathbf{C H}_{3} \text {, as } \\
\text { amplitude }\end{array}$ & $\begin{array}{l}\text { Fermi } \\
\text { amplitude }\end{array}$ & $\begin{array}{l}\text { NR } \\
\text { amplitude }\end{array}$ \\
\hline 20 & 7.71 & -5.88 & 0.05 & 0.02 \\
\hline 30 & 7.17 & -5.43 & 0.44 & 0.03 \\
\hline 40 & 6.77 & -4.85 & 1.15 & 0.03 \\
\hline 50 & 6.58 & -4.89 & 2.28 & 0.00 \\
\hline 60 & 6.89 & -4.32 & 1.23 & 0.00 \\
\hline 70 & 6.87 & -3.03 & 2.19 & 0.00 \\
\hline
\end{tabular}

Table S4: Fit amplitudes for PDMS- $\mathrm{D}_{2} \mathrm{O}$ SPS spectra (shown in Figure 2d).

\begin{tabular}{|l|l|l|l|l|}
\hline $\begin{array}{l}\text { Temperature } \\
{ }^{\circ} \mathbf{C}\end{array}$ & $\begin{array}{l}\mathbf{C H}_{3} \text { Ss } \\
\text { amplitude }\end{array}$ & $\begin{array}{l}\mathrm{CH}_{3} \text {, as } \\
\text { amplitude }\end{array}$ & $\begin{array}{l}\text { Fermi } \\
\text { amplitude }\end{array}$ & $\begin{array}{l}\text { NR } \\
\text { amplitude }\end{array}$ \\
\hline 20 & 0.23 & 4.13 & 0.00 & 0.08 \\
\hline 30 & 0.30 & 4.35 & 0.00 & 0.10 \\
\hline 40 & 0.43 & 4.58 & 0.00 & 0.11 \\
\hline 50 & 0.20 & 4.57 & 0.00 & 0.11 \\
\hline 60 & 0.13 & 4.42 & 0.00 & 0.10 \\
\hline 70 & 0.22 & 4.52 & 0.00 & 0.07 \\
\hline
\end{tabular}

\section{S3 Determining the phase of the $\mathrm{CH}_{3}$ asymmetric stretching mode in SPS polarization}

The relative phase of the $\mathrm{CH}_{3}$ symmetric and asymmetric stretching modes in the SSP spectra was obtained through fitting. However, the relative phase of the $\mathrm{CH} 3$ symmetric stretching mode in SPS and SSP still needs to be addressed. Since the ratio $A_{\mathrm{ss}, y y z} / A_{\mathrm{as}, y y z}$ is sensitive to the widths of the modes in the fitting, it is not used in the orientation analysis of the methyl groups, but it can used to determine the phase of the $\mathrm{CH}_{3}$ asymmetric stretching mode in SPS. ${ }^{14}$ For example, the spectra of the PDMS- $\mathrm{D}_{2} \mathrm{O}$ interface at $70{ }^{\circ} \mathrm{C}$ yields: $A_{\mathrm{ss}, y y z}=6.87, A_{\mathrm{as}, y y z}=-3.03$, and $A_{\mathrm{as}, y z y}= \pm 4.52$. Therefore, $A_{\mathrm{ss}, y y z} / A_{\mathrm{as}, y y z}=-2.27$, and $A_{\mathrm{as}, y y z} / A_{\mathrm{as}, y z y}= \pm 0.67$. All possible solutions of tilt and twist angles obtained from these values are presented in Figure $\mathrm{S} 1$. As shown here, $A_{\mathrm{ss}, y y z} / A_{\mathrm{as}, y y z}=-2.27$ (blue) and $A_{\text {as }, y y z} / A_{\text {as }, y z y}=-0.67$ (green) gave a similar solution for the methyl orientation (blue and green colors difficult to distinguish as they are overlapped), while $A_{\mathrm{as}, y y z} / A_{\mathrm{as}, y z y}=+0.67$ 


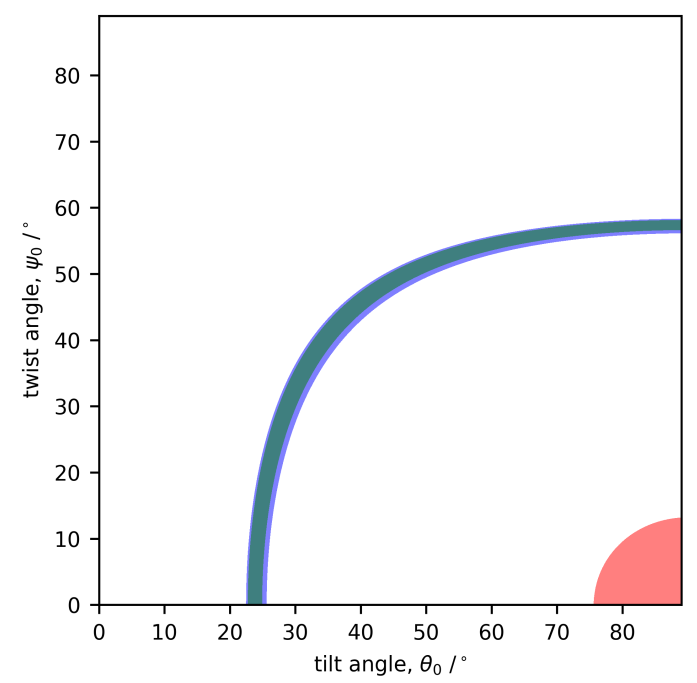

Figure S1: Orientation range obtained by: $A_{\mathrm{ss}, y y z} / A_{\mathrm{as}, y y z}=-2.27$ (blue), $A_{\mathrm{as}, y y z} / A_{\mathrm{as}, y z y}=$ -0.67 (green), and $A_{\mathrm{as}, y y z} / A_{\mathrm{as}, y z y}=+0.67$ (red) that are within $10 \%$ of the values found in the fitting of the experimental data. The blue and green regions appear blended as they are nearly perfectly overlapped.

(red) gave a solution in a vastly different region. Therefore, it is determined that $A_{\mathrm{as}, y z y}>0$.

\section{S4 Search result for temperature-dependent change in the width of the tilt and twist distribution, for a fixed value of the mean tilt and twist angle}

The resulting $A_{\text {as }, y y z} / A_{\text {as }, y z y}$ ratio and relative amplitudes of the three $\mathrm{CH}_{3}$ modes from the search as described in methods section, together with the experimental data are presented in Figure S2. Here the tilt and twist angles have a Gaussian distribution about their mean values and their widths $\sigma_{\theta}$ and $\sigma_{\psi}$ considered to be a function of temperature. As shown in the figure, even though the calculated $A_{\mathrm{as}, y y z} / A_{\mathrm{as}, y z y}$ ratio is consistent with the experimental results, the calculated relative amplitudes of the three $\mathrm{CH}_{3}$ modes are all drastically different from the experimental results, indicating that such solutions are not consistent with the experimental observations.

\section{S5 Spectra obtained upon cooling}

SFG spectra were also recorded for the PDMS-liquid interface with decreasing temperature, starting at $70{ }^{\circ} \mathrm{C}$ and cooling down to $20^{\circ} \mathrm{C}$. The spectra are presented in Figure S3. 

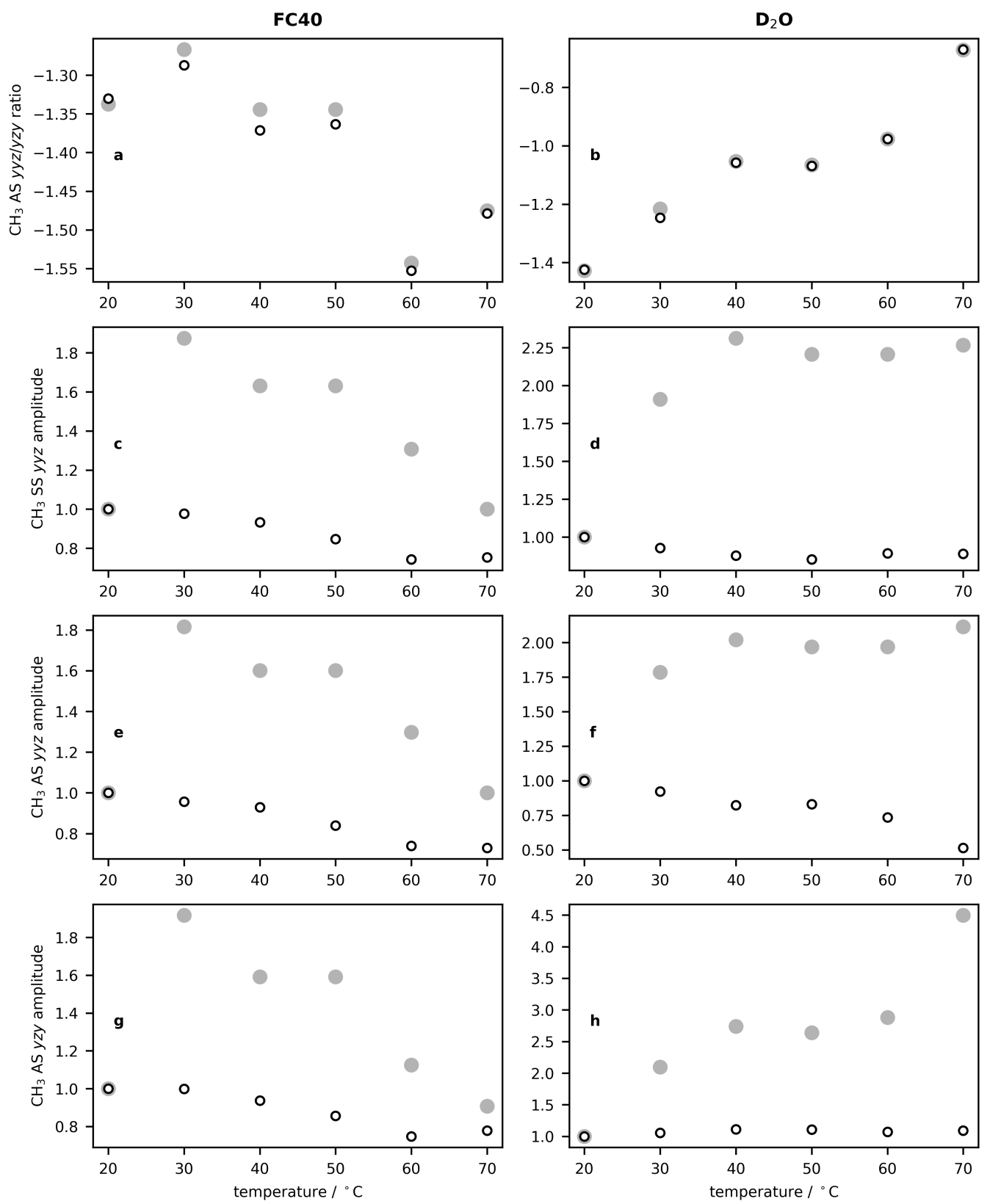

Figure S2: Fit (open black circles) and calculated (grey circles) (a, b) ratios and (c-h) amplitudes of the symmetric and asymmetric modes in SSP and SPS polarization for FC40 and $\mathrm{D}_{2} \mathrm{O}$ as a function of temperature, all normalized to $20^{\circ} \mathrm{C}$. 

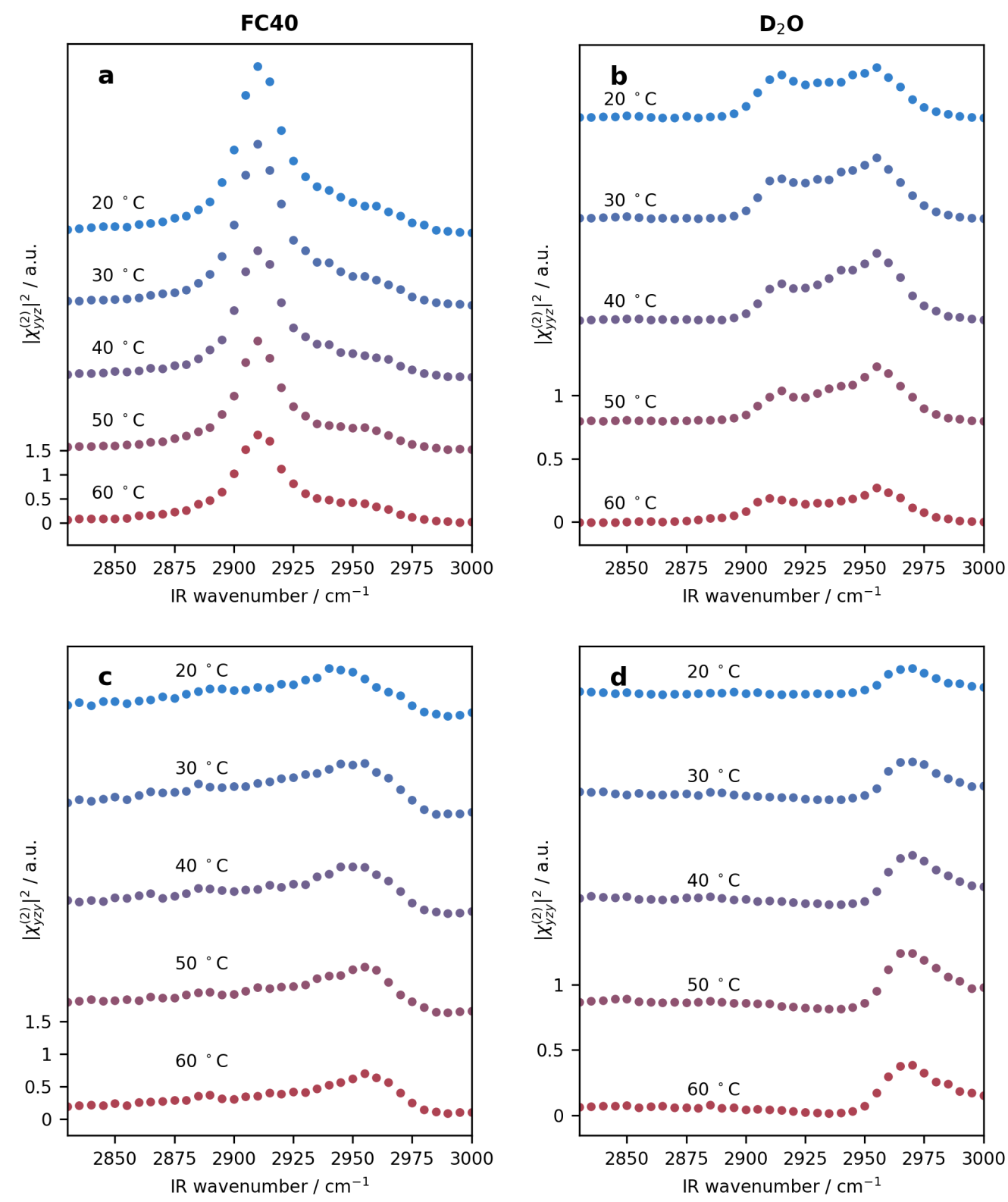

Figure S3: SFG spectra of the PDMS surface in contact with FC40 (a \& c) and $\mathrm{D}_{2} \mathrm{O}$ (b \& d) with decreasing temperature in both SSP $(a \& b)$ and SPS (c \& d) polarization schemes. The spectra at $70{ }^{\circ} \mathrm{C}$ are shown in Figure 2. Spectra are vertically offset for clarity. 


\section{References}

[1] Azam, M. S.; Cai, C.; Hore, D. K. J. Phys. Chem. C 2019, 123, 23535-23544.

[2] Cai, C.; Azam, M. S.; Hore, D. K. J. Phys. Chem. C 2021, 125, 12382-12389.

[3] Odhner, H.; Jacobs, D. J. Chem. Eng. Data 2012, 57, 166-168.

[4] Frontas' Ev, V.; Shraiber, L. J. Struct. Chem. 1965, 6, 493-500.

[5] Kedenburg, S.; Vieweg, M.; Gissibl, T.; Giessen, H. Opt. Mater. Express 2012, 2, $1588-1611$.

[6] Max, J.-J.; Chapados, C. J. Chem. Phys. 2009, 131, 184505.

[7] Matsuoka, J.; Kitamura, N.; Fujinaga, S.; Kitaoka, T.; Yamashita, H. J. Non-Cryst. Solids 1991, 135, 86-89.

[8] Looyenga, H. J. Polym Sci. 1973, 11, 1331-1336.

[9] Roberts, C. C.; Graham, A.; Nemer, M.; Phinney, L. M.; Garcia, R. M.; Soehnel, M. M.; Stirrup, E. K. Physical Properties of Low-Molecular Weight Polydimethylsiloxane Fluids. In ; Sandia National Lab: United States, 2017.

[10] Zhang, X.; Qiu, J.; Li, X.; Zhao, J.; Liu, L. Appl. Opt. 2020, 59, 2337-2344.

[11] Zhang, X.; Qiu, J.; Zhao, J.; Li, X.; Liu, L. J. Quant. Spectrosc. Radiat. Transfer 2020, 252, 107063.

[12] Electronics Materials Solutions Division, "3M Fluorinert Electronic Liquid FC-40", Technical Report, 3M, 2019.

[13] Chen, C.; Wang, J.; Chen, Z. Langmuir 2004, 20, 10186-10193.

[14] Zhang, C.; Chen, Z. J. Phys. Chem. C 2013, 117, 3903-3914.

[15] Shi, Q.; Ye, S.; Spanninga, S. A.; Su, Y.; Jiang, Z.; Chen, Z. Soft Matter 2009, 18, 3487-3494.

[16] Liu, Y.; Leng, C.; Chisholm, B.; Stafslien, S.; Majumdar, P.; Chen, Z. Langmuir 2013, 29, 2897-2905. 